\title{
The stability of a two-dimensional vorticity filament under uniform strain
}

\author{
Article \\ Published Version
}

Dritschel, D. G., Haynes, P. H., Juckes, M. N. and Shepherd, T. G. (1991) The stability of a two-dimensional vorticity filament under uniform strain. Journal of Fluid Mechanics, 230 (1). pp. 647-665. ISSN 0022-1120 doi: https://doi.org/10.1017/S0022112091000915 Available at https://centaur.reading.ac.uk/32974/

It is advisable to refer to the publisher's version if you intend to cite from the work. See Guidance on citing.

Published version at: http://dx.doi.org/10.1017/S0022112091000915

To link to this article DOI: http://dx.doi.org/10.1017/S0022112091000915

Publisher: Cambridge University Press

All outputs in CentAUR are protected by Intellectual Property Rights law, including copyright law. Copyright and IPR is retained by the creators or other copyright holders. Terms and conditions for use of this material are defined in the End User Agreement.

\section{www.reading.ac.uk/centaur}

\section{CentAUR}

Central Archive at the University of Reading 
Reading's research outputs online 


\title{
The stability of a two-dimensional vorticity filament under uniform strain
}

\author{
By D. G. DRITSCHEL, P. H. HAYNES, M. N. JUCKES $†$ \\ AND T. G. SHEPHERD
}

Department of Applied Mathematics and Theoretical Physics, University of Cambridge, Silver Street, Cambridge CB3 9EW, UK

(Received 16 February 1990 and in revised form 15 December 1990)

The quantitative effects of uniform strain and background rotation on the stability of a strip of constant vorticity (a simple shear layer) are examined. The thickness of the strip decreases in time under the strain, so it is necessary to formulate the linear stability analysis for a time-dependent basic flow. The results show that even a strain rate $\gamma$ (scaled with the vorticity of the strip) as small as 0.25 suppresses the conventional Rayleigh shear instability mechanism, in the sense that the r.m.s. wave steepness cannot amplify by more than a certain factor, and must eventually decay. For $\gamma<0.25$ the amplification factor increases as $\gamma$ decreases; however, it is only 3 when $\gamma \doteq 0.065$. Numerical simulations confirm the predictions of linear theory at small steepness and predict a threshold value necessary for the formation of coherent vortices. The results help to explain the impression from numerous simulations of two-dimensional turbulence reported in the literature that filaments of vorticity infrequently roll up into vortices. The stabilization effect may be expected to extend to two- and three-dimensional quasi-geostrophic flows.

\section{Introduction}

A ubiquitous feature of strongly nonlinear, high-Reynolds-number two-dimensional flows is the presence of thin filaments of vorticity. Considered in isolation, such filaments are unstable by the conventional Rayleigh mechanism, and would be expected to roll up into discrete vortices. However, it is a striking aspect of many high-resolution numerical experiments (e.g. McWilliams 1984; Babiano et al. 1987; Juckes \& McIntyre 1987; Brachet et al. 1988; Legras, Santangelo \& Benzi 1988; Santangelo, Benzi \& Legras 1989) that such behaviour is actually rather rare. The majority of thin filaments evolve passively under the influence of the large-scale flow (which is frequently dominated by a set of coherent vortices occupying a small fraction of the fluid domain). In this paper we consider an idealized problem that gives quantitative insight into this behaviour, wherein the large-scale flow is represented by a uniform strain field, and the consequent effects on the evolution of disturbances to a straight vorticity filament are studied. (The problem under consideration is to be distinguished from that studied by Moore \& Saffman (1975) and Tsai \& Widnall (1976) concerning the three-dimensional instability of a vortex tube in a strain field acting in the plane perpendicular to the axis of the tube. In that case the basic state is steady, the cross-section of the tube having adjusted to the strain by a suitable elongation.)

$\dagger$ Present affiliation: Department of Meteorology, University of Reading, RG6 2AU, UK.

$\ddagger$ Present affiliation: Department of Physics, University of Toronto, Toronto M5S 1A7, Canada. 


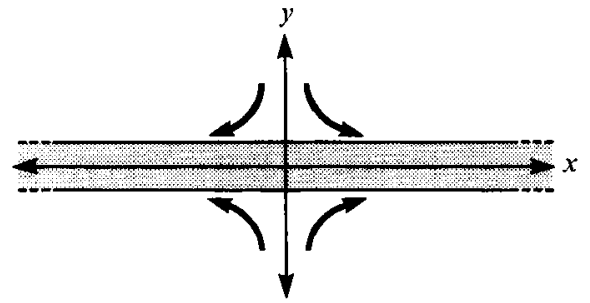

Figure 1. The simplest flow to be considered, in which a vorticity filament undergoes pure strain $(\Omega=0)$ whilst aligned parallel to the extensional axis $\left(\phi=\phi_{0}=0\right)$.

The vortex-sheet limit, where the thickness of the filament is regarded as much less than the wavelength of any disturbances, has been studied by Moore \& Griffith-Jones (1974) and Moore (1976). For fixed initial disturbance wavelength these analyses predict finite amplification. In this sense strain stabilizes. However, the predicted amplification increases without bound as the initial wavelength tends to zero. This is an artifact of the vortex-sheet model, as verified by Dhanak (1981), who later examined the qualitative effects of finite thickness in the asymptotic limit of weak strain. In the present study we present quantitative results for finite thickness with no assumption of weak strain, and we include the important additional effect of background rotation. In fact the results differ appreciably from the asymptotic results even for values of the strain rate as small as $1 \%$ of the vorticity contrast.

The simplest relevant physical situation is depicted in figure 1 . It is well known that, without strain, a strip of vorticity is unstable (Rayleigh 1880). Small undulations of the boundary grow exponentially provided that the wavelength is greater than about five times the width of the strip. The effect of strain may be seen to be two-fold. First, it compresses the disturbance in the transverse direction, thereby reducing the growth rate; and second, it extends the disturbance in the longitudinal direction, causing its wavelength to increase with time. Since the growth rates in the linearized, unstrained problem tend to zero as the wavelength tends to infinity, one expects that, ultimately, this second effect causes the disturbances, at least those of small amplitude, to decay. However, there exists the possibility of transient growth before the inevitable asymptotic decay, and it is of interest to determine the maximum growth as a function of the ratio of strain rate to vorticity contrast.

Implicit in the above is the assumption that the background strain field experienced by the filament be constant in time. It is clear that this is an oversimplification. For example, a vorticity filament in the irrotational flow outside a coherent vortex would be advected around the vortex, and so would see a rotating strain field. In a frame of reference rotating with the strain, this situation is simply a generalization of the above problem to include a uniform background vorticity. (The case where the filament is aligned along the streamlines and is not undergoing stretching was first studied by Rayleigh 1887 and nonlinear aspects of this problem have been examined by Dritschel 1989a.) Another generalization is to allow the initial orientation of the filament to lie at an angle to the extensional strain axis; the strip is subsequently rotated towards this axis.

The plan of the paper is as follows. The linearized form of the governing equations for the evolution of disturbances to a strip of uniform vorticity is derived in $\$ 2$. Each Fourier component of the disturbance can be represented by two real numbers which are the solutions of two coupled first-order linear differential equations with nonconstant coefficients. For finite strain, these equations do not appear to have a 
closed-form analytical solution and are solved numerically. The solutions presented in $\$ 3$, for the case of pure strain, confirm the qualitative picture presented above, including the existence of a period of temporary growth when the strain is sufficiently weak. The effect of rotation on the results is considered in $\$ 4$. The possibility arises that the disturbances may achieve sufficient amplitude to enter a regime in which the linear theory, and therefore the prediction of ultimate decay, becomes invalid. In particular it is possible that the strip may roll up into vortices that can resist the strain field in the manner described by Moore \& Saffman (1971) and Kida (1981), rather than being torn apart. This possibility motivates numerical integration of the fully nonlinear equations in $\$ 5$ using a contour dynamics algorithm. Calculations show that the initial wave slopes necessary for vortex roll-up are numerically small.

\section{Mathematical formulation}

The basic state consists of an infinitely long strip of uniform vorticity $\omega$ and thickness $D(t)$ orientated at an angle $\phi(t)$ with respect to the $x$-axis (see figure 2), in the presence of a general linear strain field

$$
u(x, y)=\gamma x-\Omega y, \quad v(x, y)=\Omega x-\gamma y,
$$

where $\gamma$ is the strain rate, and $2 \Omega$ the background vorticity, both constants. This is equivalent to an irrotational strain field of strength $\gamma$ rotating at a rate $-\Omega$. The equations are non-dimensionalized so that the anomalous vorticity of the strip, $\omega$, and its initial width, $D(0)$, are both equal to unity. There is no loss of generality in taking $\gamma$ to be positive.

It is not difficult to show that this strain field causes the orientation $\phi$ and thickness $D$ of the strip to vary with time according to

$$
\frac{\mathrm{d} \phi}{\mathrm{d} t} \equiv \dot{\phi}=\Omega-\gamma \sin 2 \phi, \quad \frac{1}{D} \frac{\mathrm{d} D}{\mathrm{~d} t}=-\gamma \cos 2 \phi
$$

(Kida 1981; Dritschel 1990), from which it follows that

$$
D^{2}(t)=\frac{\Omega-\gamma \sin 2 \phi}{\Omega-\gamma \sin 2 \phi_{0}},
$$

where $\phi_{0}=\phi(0)$. Note that in the special case $\Omega=0$ and $\phi_{0}=0$ depicted in figure 1 , (3) is singular and $(2 b)$ gives simply $D=\exp (-\gamma t)$. In general, if $\gamma \geqslant|\Omega|$, then $(2 a)$ shows that ultimately $\phi$ approaches the extensional axis $\phi_{\mathrm{c}} \equiv \frac{1}{2} \sin ^{-1}(\Omega / \gamma)$. The value of $\sin ^{-1}$ is taken to lie between $-\frac{1}{2} \pi$ and $+\frac{1}{2} \pi$. Equation $(2 b)$ consequently implies $D(t) \sim \exp \left(-\left(\gamma^{2}-\Omega^{2}\right)^{\frac{1}{2}} t\right)$ as $t \rightarrow \infty$. On the other hand, if $\gamma<|\Omega|$, the strip will thin and thicken periodically. In this paper we restrict our attention to extending (and thinning) strips, but note that the stability of the periodic case can be studied using Floquet theory (see Dritschel 1990 for details).

The linear disturbance equations are derived as follows. We employ the coordinate system shown in figure 2 , rotating at angular velocity $\dot{\phi}$ as given by $(2 a)$, in which the strip is always parallel to the $x^{\prime}$-axis. In these coordinates, the velocity $\left(u^{\prime}, v^{\prime}\right)$ is given by

$$
\begin{aligned}
u^{\prime} & =\gamma\left(x^{\prime} \cos 2 \phi-y^{\prime} \sin 2 \phi\right)+(\dot{\phi}-\Omega) y^{\prime}+u_{\mathrm{s}}^{\prime} \\
& =\gamma\left(x^{\prime} \cos 2 \phi-2 y^{\prime} \sin 2 \phi\right)+u_{\mathrm{s}}^{\prime} \\
v^{\prime} & =-\gamma\left(y^{\prime} \cos 2 \phi+x^{\prime} \sin 2 \phi\right)-(\dot{\phi}-\Omega) x^{\prime}+v_{\mathrm{s}}^{\prime} \\
& =-\gamma y^{\prime} \cos 2 \phi+v_{\mathrm{s}}^{\prime},
\end{aligned}
$$

where $\left(u_{\mathrm{s}}^{\prime}, v_{\mathrm{s}}^{\prime}\right)$ is the velocity associated with the strip of vorticity. 


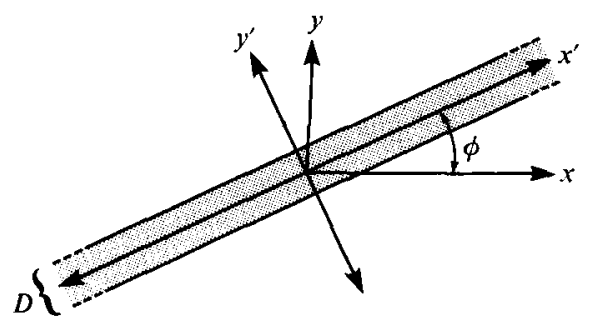

Figure 2. Diagram of a general flow configuration, with the filament aligned at an arbitrary angle $\phi \neq 0$ to the extensional axis, defining the primed coordinate system that moves with the filament.

For the undisturbed strip we have that $\left(u_{\mathrm{s}}^{\prime}, v_{\mathrm{s}}^{\prime}\right)=\left(u_{\mathrm{s}}\left(y^{\prime}\right), 0\right)$, where

$$
u_{\mathrm{s}}\left(y^{\prime}\right) \equiv\left\{\begin{array}{cll}
-\frac{1}{2} D(t) & \text { if } & y^{\prime}>\frac{1}{2} D(t), \\
-y^{\prime} & \text { if } & \left|y^{\prime}\right| \leqslant \frac{1}{2} D(t), \\
\frac{1}{2} D(t) & \text { if } & y^{\prime}<-\frac{1}{2} D(t) .
\end{array}\right.
$$

It is evident from (4) and (5) that the undisturbed flow is a combination of pure strain $\gamma \cos 2 \phi$ plus a piecewise-linear shear flow in the $x^{\prime}$-direction.

The vorticity of the flow being piecewise constant, the velocity field $u_{\mathrm{s}}^{\prime}$, $v_{\mathrm{s}}^{\prime}$ is determined completely by the positions of the two edges of the strip, and, using the standard Green function for the Laplacian operator, may be written in the form

$$
\left(u_{\mathrm{s}}^{\prime}\left(x^{\prime}, y^{\prime}\right), v_{\mathrm{s}}^{\prime}\left(x^{\prime}, y^{\prime}\right)\right)=\frac{1}{4 \pi} \int_{\mathscr{C}} \log \left\{\left(x^{\prime}-\xi\right)^{2}+\left(y^{\prime}-\zeta\right)^{2}\right\}\langle\mathrm{d} \xi, \mathrm{d} \zeta)
$$

where $\mathscr{C}$ is the contour comprising the boundaries of the strip, traversed in such a way that the interior of the strip is on the left, and $(\mathrm{d} \xi, \mathrm{d} \zeta)$ is the vector tangent to the boundary. The boundaries of the strip are denoted by $y^{\prime}=y_{ \pm}^{\prime}\left(x^{\prime}, t^{\prime}\right)$ and, noting that $y_{ \pm}^{\prime}$ will be expected to scale with $D(t)$, we define the disturbance $\eta_{ \pm}\left(x^{\prime}, t^{\prime}\right)$ by

$$
y_{ \pm}^{\prime}\left(x^{\prime}, t^{\prime}\right)=D(t)\left( \pm \frac{1}{2}+\epsilon \eta_{ \pm}\left(x^{\prime}, t\right)\right)
$$

where $\epsilon$ is a small parameter. We consider the evolution of disturbances of the form

$$
\eta_{ \pm}=\operatorname{Re}\left\{\hat{\eta}_{ \pm}(t) \exp \left(\mathrm{i} k D(t) x^{\prime}\right)\right\}
$$

where $k$ is a constant equal to the initial wavenumber and the factor $D$ reflects the stretching in the $x^{\prime}$ direction. Substituting (7) into (6), using the kinematic boundary condition

$$
\frac{\partial y_{ \pm}^{\prime}}{\partial t}+u^{\prime}\left(x^{\prime}, y_{ \pm}^{\prime}, t\right) \frac{\partial y_{ \pm}^{\prime}}{\partial x}=v^{\prime}\left(x, y_{ \pm}^{\prime}, t\right)
$$

and isolating $O(\epsilon)$ terms we arrive at the linearized governing equations,

$$
\mathrm{i} \frac{\mathrm{d} \hat{\eta}_{ \pm}}{\mathrm{d} t}= \pm \frac{1}{2}[1-k \Delta(1-\Lambda)] \hat{\eta}_{ \pm} \mp \frac{1}{2} \mathrm{e}^{-k \Delta} \hat{\eta}_{\mp}
$$

where

$$
\Delta \equiv D^{2}(t)
$$

and $A \equiv-2 \gamma \sin 2 \phi$ is the instantaneous value of the shear measured in a frame rotating with the strip. These equations may also be derived by direct consideration of a boundary-value problem for the disturbance following Rayleigh (1880); and the extension to single- and multi-layer quasi-geostrophic flows is possible by either method. They are precisely equivalent to Rayleigh's equations except that $\Delta$ and $A$ may vary with time and it may therefore not be assumed that growth or decay is exponential. 
It is advantageous to rewrite (10) in terms of the quantities $A=\hat{\eta}_{+}+\hat{\eta}_{-}$and $B=$ $\mathrm{i}\left(\hat{\eta}_{+}-\hat{\eta}_{-}\right)$, for then the resulting equations have real coefficients :

$$
\begin{aligned}
& \frac{\mathrm{d} A}{\mathrm{~d} t}=-\frac{1}{2}\left[1-k \Delta(1-\Lambda)+\mathrm{e}^{-k \Delta}\right] B, \\
& \frac{\mathrm{d} B}{\mathrm{~d} t}=+\frac{1}{2}\left[1-k \Delta(1-\Lambda)-\mathrm{e}^{-k \Delta}\right] A .
\end{aligned}
$$

We have been unable to find closed-form analytic solutions to $(11 a)$ and $(11 b)$ and have resorted to numerical solution. Although analytical progress can be made in the small-strain limit (see §3) the results seem to be of limited usefulness.

The quantity of interest is the amplification maximized over initial wavenumber $k$, and its variation with the initial orientation $\phi_{0}$, the strain rate $\gamma$ and the rotation rate $\Omega$. We measure amplification by the increase in the r.m.s. wave slope

$$
\left\{\frac{k D}{2 \pi} \int_{0}^{\frac{2 \pi}{k D}} \mathrm{~d} x^{\prime}\left(\left(\frac{\partial y_{+}^{\prime}}{\partial x^{\prime}}\right)^{2}+\left(\frac{\partial y_{-}^{\prime}}{\partial x^{\prime}}\right)^{2}\right)\right\}^{\frac{1}{2}} .
$$

The square of this quantity is proportional to the relative size of the nonlinear terms neglected in the derivation of (10). (Note that the validity of the linear theory requires only that the wave slope be small; the displacements need not necessarily be small compared to the width of the strip.) Evaluating (12) with $|A|^{2}+|B|^{2}=1$ at $t=0$ (recall that $\Delta=1$ at $t=0$ as well) and taking the square root then gives the corresponding amplification factor

$$
\mathscr{A}=\Delta\left(|A|^{2}+|B|^{2}\right)^{\frac{1}{2}} .
$$

The disturbance energy is proportional to $\Delta^{\frac{1}{2}}\left(|A|^{2}+|B|^{2}\right)$. However, this measure of amplification has a less direct relationship with the importance of nonlinearity.

Note that for stretching strips, $\Delta(t)$ in (13) diminishes with time. Two effects of the external strain field are contributing to this. The first is simply the thinning of the width of the layer, and this contributes a factor $D=\Delta^{\frac{1}{2}}$. The second is the stretching of the disturbance wavelength as the strip stretches at the same rate, and this contributes another factor of $D$. Both effects tend to work against wave-slope amplification.

$\mathscr{A}$ as defined by (13) depends on $A(0)$ and $B(0)$ besides $k, \phi_{0}, \gamma, \Omega$ and $t$. The first two of these parameters can be eliminated by searching for the form of the initial perturbation that, by a given time $t$, has amplified the most. Because the two evolution equations are linear, any solution $(A, B)$ can be expressed in terms of two independent solutions, say $\left(A_{1}, B_{1}\right)$ and $\left(A_{2}, B_{2}\right)$ :

$$
\begin{aligned}
& A(t)=c_{1} A_{1}(t)+c_{2} A_{2}(t), \\
& B(t)=c_{1} B_{1}(t)+c_{2} B_{2}(t),
\end{aligned}
$$

where $c_{1}$ and $c_{2}$ are complex numbers. The two solutions are made independent by choosing different initial conditions for them. A suitable choice is

$$
\begin{array}{ll}
A_{1}(0)=1, & B_{1}(0)=0, \\
A_{2}(0)=0, & B_{2}(0)=1,
\end{array}
$$

in which case $A(0)=c_{1}$ and $B(0)=c_{2}$. Putting $c_{1}=\mathrm{e}^{\mathrm{i} \lambda_{1}} \cos \theta$ and $c_{2}=\mathrm{e}^{\mathrm{i} \lambda_{2}} \sin \theta$ (to satisfy $|A(0)|^{2}+|B(0)|^{2}=1$ ), one simply maximizes $|A(t)|^{2}+|B(t)|^{2}$ with respect to $\theta$ and $\lambda \equiv \lambda_{2}-\lambda_{1}$. In terms of the quantities $\mathscr{A}_{1}^{2} \equiv \frac{1}{2}\left(A_{1}^{2}+B_{1}^{2}\right), \mathscr{A}_{2}^{2} \equiv \frac{1}{2}\left(A_{2}^{2}+B_{2}^{2}\right)$ and 
$\mathscr{B} \equiv A_{1} A_{2}+B_{2} B_{2}$, it is straightforward to show that the most that a disturbance can amplify by time $t$ is given by

$$
\overline{\mathscr{A}}\left(k, \phi_{0}, \gamma, \Omega, t\right) \equiv \Delta\left(\left(\mathscr{A}_{1}^{2}+\mathscr{A}_{2}^{2}\right)+\left[\left(\mathscr{A}_{2}^{2}-\mathscr{A}_{1}^{2}\right)^{2}+\mathscr{B}^{2}\right]^{\frac{1}{2}}\right)^{\frac{1}{2}} .
$$

(This result depends only on the definition of $\mathscr{A}$ and is independent of the specific form of the equations examined in this paper.) In order to determine $\bar{A}$ one then has only to solve the evolution equations for the two initial conditions $\left(A_{1}, B_{1}\right)$ and $\left(A_{2}\right.$, $B_{2}$ ).

Finally, maximizing $\overline{\mathscr{A}}$ over time $t$ and initial wavenumber $k$ gives the required quantity $\mathscr{A}_{\mathrm{m}}\left(\phi_{0}, \gamma, \Omega\right)$. For reference, we define $t_{\mathrm{m}}\left(\phi_{0}, \gamma, \Omega\right)$ and $k_{\mathrm{m}}\left(\phi_{0}, \gamma, \Omega\right)$ respectively as the time to reach maximum amplification and the initial dimensionless wavenumber for which this maximum is achieved (i.e. $\mathscr{A}_{\mathrm{m}}\left(\phi_{0}, \gamma, \Omega\right)=$ $\left.\bar{A}\left(k_{\mathrm{m}}, \phi_{0}, \gamma, \Omega, t_{\mathrm{m}}\right)\right)$. Also, we define $\varphi_{\mathrm{m}}\left(\phi_{0}, \gamma, \Omega\right)$ as the initial phase difference between the waves on the two interfaces for the optimal solution, namely $\varphi_{\mathrm{m}} \equiv$ $\varphi_{-}-\varphi_{+}$where $\eta_{ \pm}=R_{ \pm} \mathrm{e}^{\mathrm{i} \varphi_{ \pm}}$. It follows from the definition of $\hat{\eta}_{ \pm}$that $\varphi_{\mathrm{m}}$ is given by $\tan ^{-1}\left[|\mathscr{B}| /\left(\mathscr{A}_{2}^{2}-\mathscr{A}_{1}^{2}\right)\right]$. Note that by symmetry, the optimal solution must have equal disturbance amplitude on both interfaces, namely $R_{+}=R_{-}$.

In the next two sections $\mathscr{A}_{m}$ and associated quantities are determined by numerical integration of (11) using the two initial conditions given in (15). The integration uses a standard fourth-order Runge-Kutta scheme with time step $\Delta t=$ 0.025 coupled with quadratic polynomial interpolation to determine the time when $\mathscr{A}$ reaches its first maximum greater than 1 . If such a maximum is not encountered before $\Delta(t)<0.01$, it is assumed that $\bar{A}$ will never become comparable to 1 again. Should a maximum be found, the integration is not carried further to check for other maxima. Tests have indicated that there is only one maximum in most cases, and if there are more than one, that the first maximum is the largest. This procedure is repeated for many wavenumbers $k$ to determine $\mathscr{A}_{m}$, i.e. $\mathscr{A}$ maximized over both time and wavenumber.

\section{A strip in pure strain}

As a first problem we consider a strip of vorticity centred on the extensional axis of strain $\left(\phi_{0}=0\right)$ in the absence of rotation $(\Omega=0)$, as shown in figure 1 . The layer thickness diminishes exponentially, $D(t)=\mathrm{e}^{-\gamma t}$, and the strip remains centred on the $x$-axis, $\phi(t)=0$.

It is useful to recall that in the unstrained problem (where $\Delta$ remains equal to 1 ) disturbances are neutrally stable for $k>k_{\mathrm{c}} \doteq 1.28$ and exponentially growing (or decaying) for $k<k_{\mathrm{c}}$, although the growth rate tends to zero as $k \rightarrow 0$. Thus, given the tendency of the strain to diminish disturbance amplitudes and to decrease wavenumbers, we might expect that for an initial condition with $k>k_{\mathrm{c}}$ a disturbance will initially decay, but then grow when the effective wavenumber $k \Delta$ reduces to values less than $k_{\mathrm{c}}$. Ultimately $k \Delta$ becomes so small that any tendency for growth is overcome by the direct effect of the straining.

A number of examples of the evolution of $\mathscr{A}$ for various cases, all having $k=$ $k_{\mathrm{m}}=1.4776$ but with a variety of initial phases, are shown in figure $3(a)$, for the case $\gamma=0.05$. Also plotted is the time variation of the amplification maximized over all initial phases, $\mathscr{A}$, again for $k=k_{\mathrm{m}}$. Note that for some of the initial conditions $\mathscr{A}$ increases initially, even though the initial wavenumber is greater than $k_{\mathrm{e}}$ (and therefore the qualitative arguments above would suggest initial decay). This is a manifestation of the temporary amplification possible in many systems, even when 

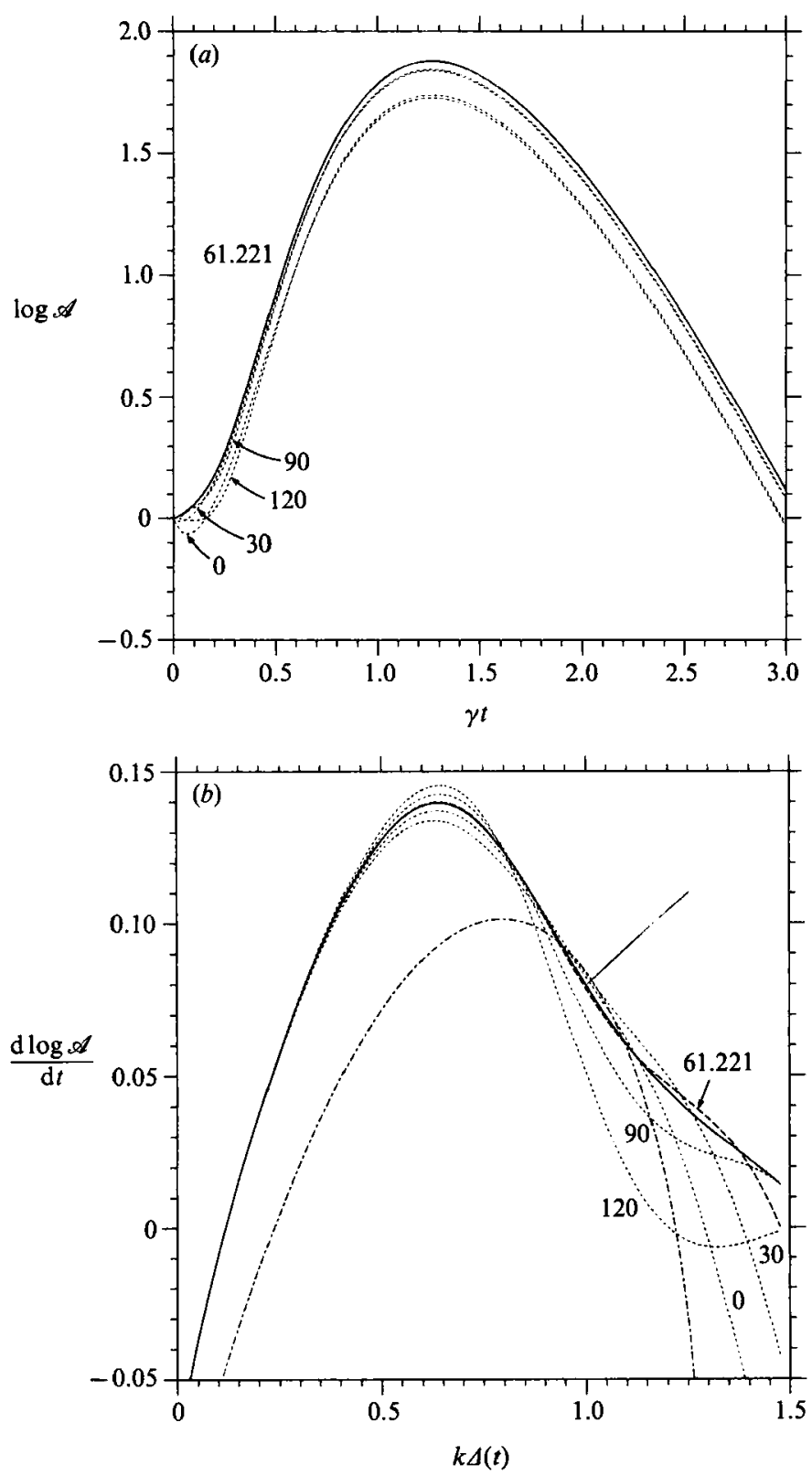

Figure 3. (a) Time evolution of the disturbance norm $\mathscr{A}$ for disturbances to an aligned strip in pure strain $(\Omega=0)$, with $\gamma=0.05$, shown as dotted curves. The disturbances have initial wavenumber equal to that which gives the maximum amplification, and various initial phases, as labelled. The solid curve shows the evolution of the maximum possible amplification (over all initial phases) $\mathscr{A}$, for this initial wavenumber. A dashed curve shows $\mathscr{A}$ for the initial phase $\varphi=\varphi_{\mathrm{m}}=61.221^{\circ}$, that leads to the maximum amplification at any time, but this curve is indistinguishable from the solid curve. (b) The same, except that the logarithmic time rate of change of the various quantities is shown and plotted against the effective wavenumber (which decreases exponentially with time). The dash-dot curve is the growth rate predicted by a quasi-steady analysis, namely the growth rate in the unstrained stability problem, minus twice the strain rate. 

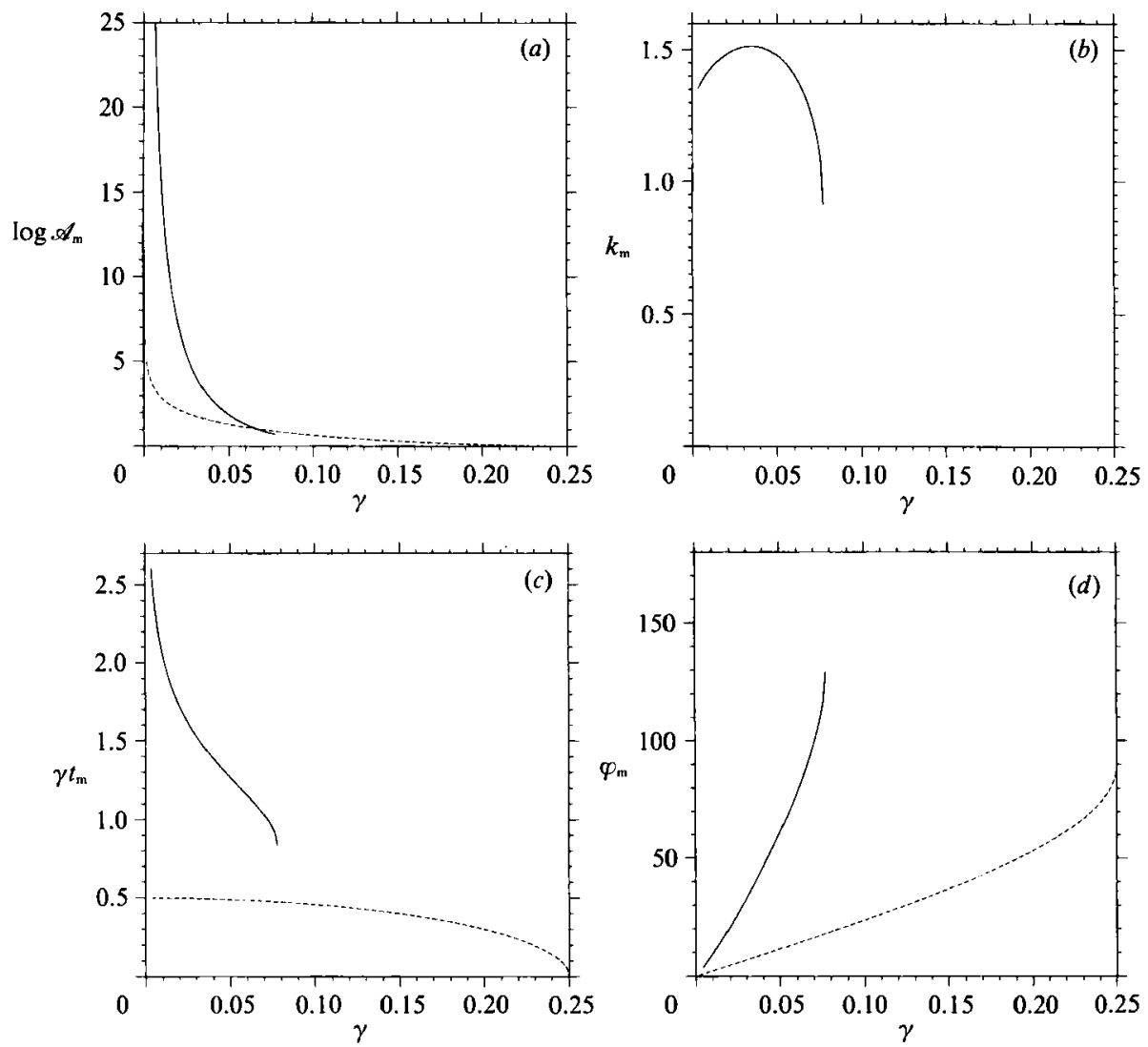

Figure 4. Results for the case of an aligned strip in pure strain $\left(\Omega=0, \phi_{0}=0\right)$. The two curves correspond to qualitatively different maximizing disturbances: the solid curve to intermediate wavelengths and the dashed curve to long wavelengths (see txt for details). (a) $\log \mathscr{A}_{\mathrm{m}} v s . \gamma ;(b) k_{\mathrm{m}}$ vs. $\gamma ;(c) \gamma t_{\mathrm{m}} v s . \gamma ;(d) \varphi_{\mathrm{m}} v s . \gamma$.

there are no exponentially growing modes. Of course, the maximum amplification factor $\mathscr{A}$ takes account of such temporary amplification. These features are apparent in figure $3(b)$, which shows, as a function of effective wavenumber $k \Delta$, the growth rate (measured by the logarithmic rate of change with time) of $\mathscr{A}$, for each of the cases shown in figure $3(a)$, and of $\mathscr{A}$. Also shown is the growth rate predicted by a quasi-steady calculation. (The coordinate $k \Delta$ plotted along the abscissa decreases exponentially with increasing time.) It is noteworthy that the growth rate is generally underestimated by the quasi-steady approximation.

Figure $4(a-d)$ shows $\log \mathscr{A}_{\mathrm{m}}, k_{\mathrm{m}}, \gamma t_{\mathrm{m}}$, and $\varphi_{\mathrm{m}}$ as a function of the strain rate $\gamma$. In each of figures $4(a), 4(c)$ and $4(d)$ there are two curves shown, each corresponding to a different amplification mode. The dashed curves correspond to the amplification of disturbances that have long wavelength $(k \ll 1)$ initially. The solid curves correspond to the amplification of waves with intermediate wavelength initially. The corresponding initial wavenumbers are shown in figure $4(b)$. The origin of these distinct modes of amplification is apparent from the variation of $\mathscr{A}$ maximized over time as a function of initial wavenumber $k$. There are found to be two maxima, one at intermediate wavelength and the other at the long-wavelength limit, $k=0$. For small $\gamma$ the value of $\mathscr{A}$ at the latter is smaller, but as $\gamma$ increases the value of $\mathscr{A}$ at intermediate wavelengths decreases, relative to that at $k=0$. For $\gamma>0.066$ the 

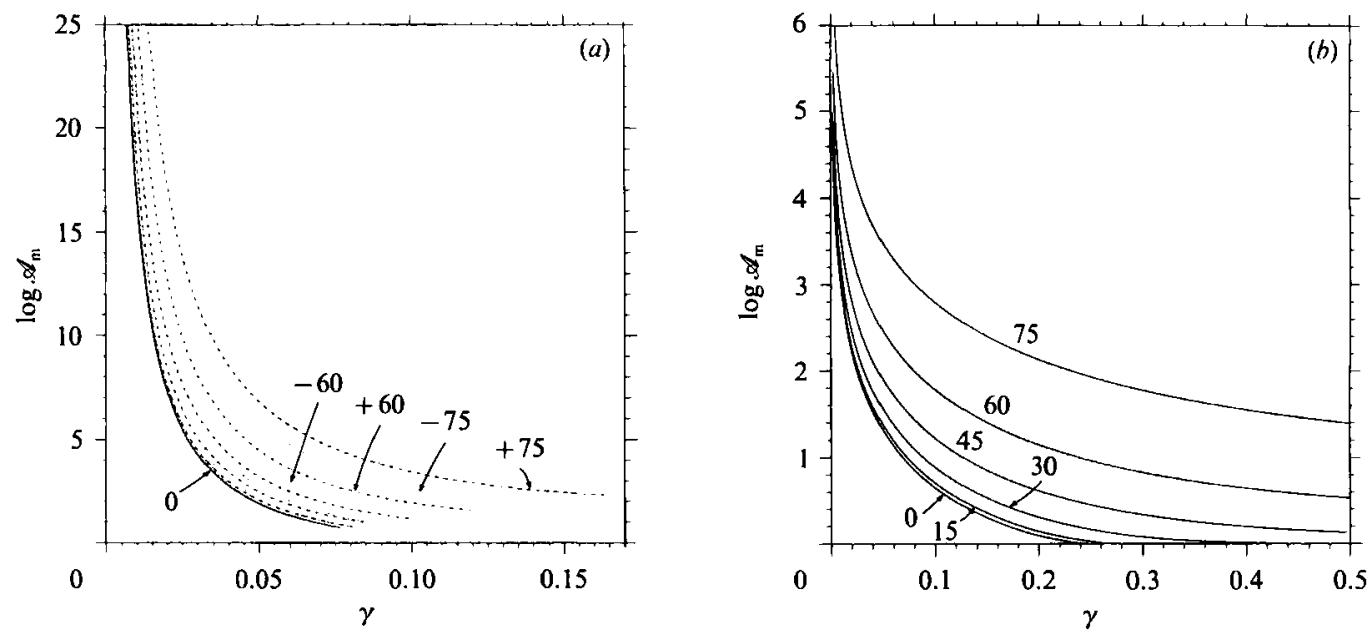

FiguRe 5. (a) $\log \mathscr{A}_{\mathrm{m}}$ for non-aligned strips in pure strain $\left(\Omega=0, \phi_{0} \neq 0\right)$. The results are given as a function of the straian rate $\gamma$, for selected initial orientation, with $0<\gamma \leqslant 0.17$ and $-80^{\circ} \leqslant$ $\phi_{0} \leqslant 80^{\circ}$ as labelled. $(b)$ The same, but for long-wave disturbances $(k \ll 1)$ with $0<\gamma \leqslant 0.5$.

maximum amplification is found in the long-wave limit analysed in Appendix A. It follows from this analysis that for $\gamma>\frac{1}{4}$, all disturbances decay.

The important implication of the results shown in figure 4 is that even weak strain can strongly inhibit the amplification of disturbances. For instance, at most a threefold amplification can occur when $\gamma=0.065$ (figure $4 a$ ). Even for $\gamma=0.05$ the maximum amplification is only 6.5461 .

On the other hand, the amplification can be quite considerable when the strain rate is very small. This is, of course, consistent with the unbounded amplification that occurs in the absence of strain. The small-strain case may be examined by WKB analysis and Dhanak (1981) has shown that the largest factor in $\mathscr{A}_{\mathrm{m}}$ takes the form $\exp (r / \gamma)$ where $r$ is a positive constant. In Appendix B (equation (B 4a)) the constant $r$ is found to be 0.188 and the asymptotic analysis is extended to give a fuller expression for $\mathscr{A}_{\mathrm{m}}$ that may be usefully compared with the numerical results. The details of the analysis in Appendix B suggest that values of $\gamma$ less than $10^{-3}$ are necessary for good agreement with the asymptotic expression. $\mathscr{A}_{\mathrm{m}}$ is then greater than $10^{81}$. For $\gamma=0.05$ the computed value of the asymptotic expression for $\mathscr{A}_{\mathrm{m}}$ is 49.7, more than seven times larger than the actual value.

A straightforward variation on the above problem is to allow the initial orientation of the strip as shown in figure 2 to be at an arbitrary angle, $\phi=\phi_{0}$ say, to the extensional axis of the strain field. In time, the strip will become progressively aligned with the $x$-axis, except in the exceptional case for which $\phi_{0}= \pm \frac{1}{2} \pi$ (where the strip remains aligned along the axis of contraction rather than extension and the strain field augments rather than inhibits the growth of disturbances). Solving (2a) directly (with $\Omega=0$ ) gives

$$
\tan \phi(t)=\mathrm{e}^{-2 \gamma t} \tan \phi_{0},
$$

while from (3) one has $\sin 2 \phi(t)=\Delta(t) \sin 2 \phi_{0}$. Put together, one finds that the squared thickness of the strip evolves in time according to

$$
\Delta(t)=\frac{1}{\mathrm{e}^{2 \gamma t} \cos ^{2} \phi_{0}+\mathrm{e}^{-2 \gamma t} \sin ^{2} \phi_{0}} .
$$

Figure $5(a)$ shows a graph of $\log \mathscr{A}_{\mathrm{m}}$ as a function of the strain rate for selected values of $\phi_{0}$ and figure $5(b)$ shows the corresponding result for long-wave disturbances. It 


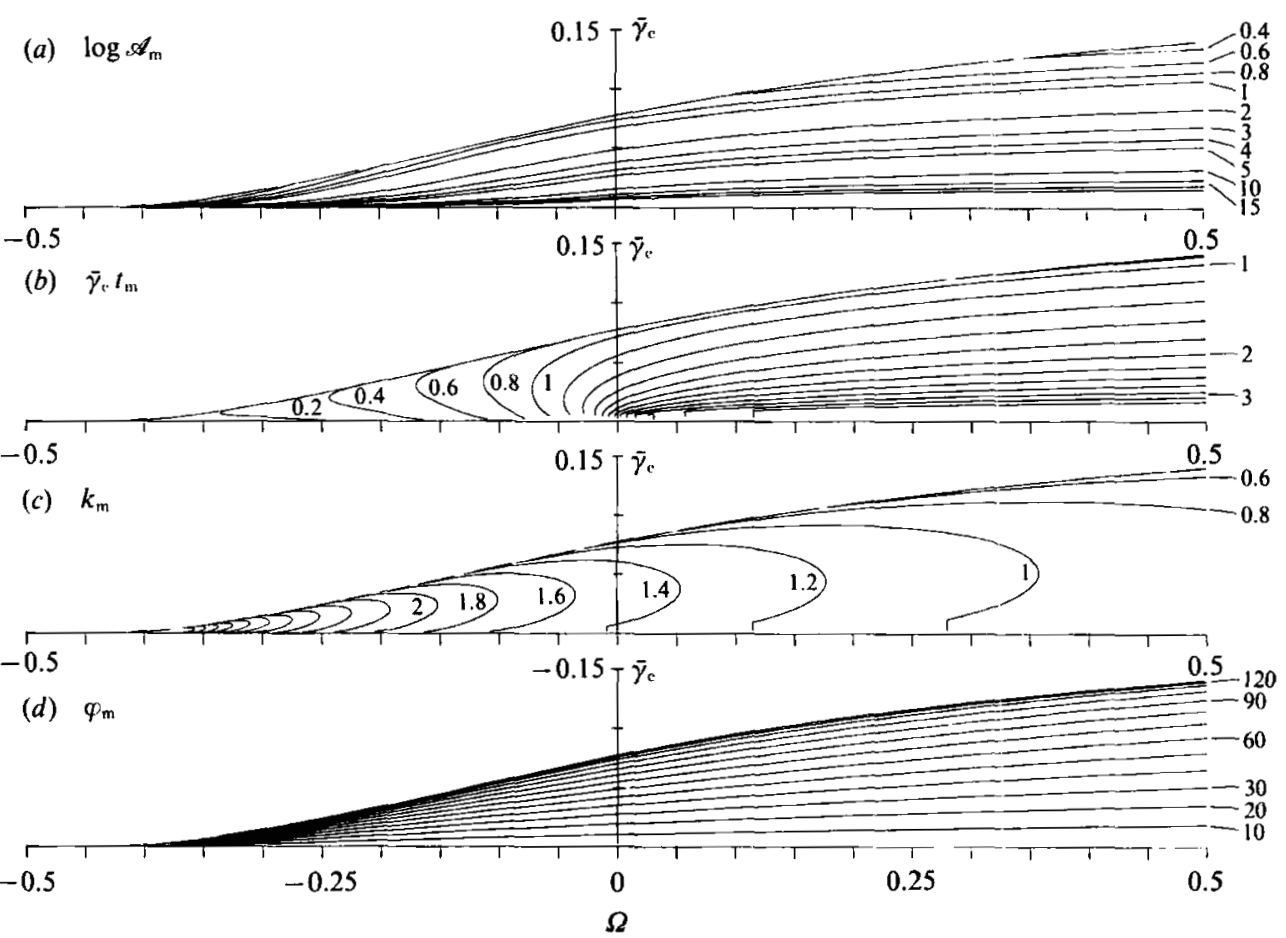

FIGURE 6. Results for aligned strips in a general strain field $\left(\phi_{0}=\phi_{\mathrm{c}}, \gamma>|\Omega|\right)$. The results are plotted as a function of $\Omega$ and the stretching rate $\bar{\gamma}_{\mathrm{c}}=\left(\gamma^{2}-\Omega^{2}\right)^{\frac{1}{2}}$, with $-0.5 \leqslant \Omega \leqslant 0.5$ and $0.01 \leqslant \bar{g}_{\mathrm{c}} \leqslant 0.25$. (a) $\log \mathscr{A}_{\mathrm{m}} ;(b) \bar{\gamma}_{\mathrm{c}} t_{\mathrm{m}} ;(c) k_{\mathrm{m}} ;(d) \varphi_{\mathrm{m}}$.

is shown in Appendix A that the boundary separating amplifying and decaying disturbances in the $\left(\gamma, \phi_{0}\right)$-plane is given by $4 \gamma \cos 2 \phi_{0}=1$. Note that the greater the initial angle $\phi_{0}$, the less the strip is stretched initially and the greater the amplification. Indeed if $\left|\phi_{0}\right|>\frac{1}{4} \pi$ the initial effect of the strain is to thicken the strip and thereby augment the growth of disturbances. The asymmetry with respect to $\phi_{0}$ derives from the term $A=-2 \gamma \sin 2 \phi$ in (11). For $A>0\left(\phi, \phi_{0}<0\right)$, this term gives rise to 'adverse shear', a known stabilizing effect in the case of non-stretching, sheared strips (Rayleigh 1887; Dritschel 1989a), while $\Lambda<0$, termed 'cooperative shear', augments instability.

\section{A strip in strain with non-zero rotation}

In this section, a more general problem is considered for which both the strain rate and the rotation rate are non-zero, but attention is restricted to $|\gamma| \geqslant|\Omega|$ so that, by $(2 a)$, the strip continually extends. We first consider a strip which is initially aligned at the equilibrium angle $\phi_{\mathrm{c}}=\frac{1}{2} \sin ^{-1}(\Omega / \gamma)$, so that $\phi$ remains equal to $\phi_{\mathrm{c}}$ for all time. The case of arbitrary initial $\phi$ is discussed briefly below.

With $\phi=\phi_{c},(3)$ is singular; but solving $(2 b)$ one sees that the thickness of the strip diminishes exponentially, at the rate $\bar{\gamma}_{\mathrm{c}} \equiv\left(\gamma^{2}-\Omega^{2}\right)^{\frac{1}{2}}=\gamma \cos 2 \phi_{\mathrm{c}}$, while the shear across the layer, $\Lambda$, is given by $\Lambda=-2 \gamma \sin 2 \phi_{\mathrm{c}}=-2 \Omega$. Both $\bar{\gamma}_{\mathrm{c}}$ and $\Lambda$ remain constant in time.

Figure $6(a-d)$ gives contour plots of $\log \mathscr{A}_{\mathrm{m}}, k_{\mathrm{m}}, \bar{\gamma}_{\mathrm{c}} t_{\mathrm{m}}$, and $\varphi_{\mathrm{m}}$ as a fucntion of $\bar{\gamma}_{\mathrm{c}}$ and $\Omega\left(=-\frac{1}{2} \Lambda\right)$. As remarked in the previous section, adverse shear $(\Omega<0)$ inhibits 
the amplification of disturbances, while cooperative shear $(\Omega>0)$ enhances it. Whatever the value of $\Omega$, increasing $\bar{\gamma}_{\mathrm{c}}$ reduces amplification. The corresponding results for long-wave disturbances are the same as already given in figure 4 (the dashed lines) with the substitution of $\bar{\gamma}_{\mathrm{c}}$ for $\gamma$. (As shown in Appendix A, the results are independent of $\Omega$.) In particular, a strain rate $\bar{\gamma}_{\mathrm{c}}$ in excess of $\frac{1}{4}$ suppresses the amplification of any disturbance.

With $\phi \neq \phi_{0}$, as in the analogous pure strain case considered in $\S 3$, the strip adjusts to the extensional axis of strain and the amplification increases with $\left|\phi-\phi_{0}\right|$ as a result.

\section{Finite-amplitude effects}

We next examine the evolution of disturbances at finite amplitude. The breakdown of the validity of the linearized form of (6) has been examined by Dritschel (1988, Appendix B) and Shelly \& Baker (1990). It appears that the linearization involved in the derivation of $(11 a)$ and (11b) breaks down when the wave slope (12) is of order unity. The linearization does not break down when the displacements are comparable to the thickness of the strip (i.e. when $\left|\eta_{ \pm}\right| \approx O(1)$ ) provided that the wave slope is still small. This is possible in the long-wavelength limit and will be discussed further below. We might then expect that the prediction of maximum wave slope on the basis of linear theory would be a useful indicator of the potential importance of finiteamplitude effects in the real flow. Here, we investigate this hypothesis by following the nonlinear evolution of a series of flows differing only in the expected maximum wave slope predicted from linear theory. We examine just the case of pure strain $(\Omega=0)$ and aligned strip orientation $\left(\phi_{0}=0\right)$, but we expect the results to be indicative of the behaviour one would see in other circumstances.

The calculations are performed using 'contour surgery', an extended and refined version of contour dynamics, on a domain which is periodic in the $x$-direction and expanding with the straining flow. Full details of the numerical method can be found in Dritschel $(1989 b)$.

The initial conditions for the calculations consist of a strip plus a disturbance which has the same periodicity as the computational domain. The initial disturbance is chosen to be the one that amplifies most in linear theory. The initial wave slope, $\beta_{0}$ say, is chosen such that the expected maximum wave slope, $\beta_{\mathrm{m}}=\mathscr{A}_{\mathrm{m}} \beta_{0}$, takes prescribed values. We fix the strain rate at $\gamma=0.05$. At this value, $\mathscr{A}_{\mathrm{m}}=6.5461$.

When $\beta_{\mathrm{m}}<0.2$, nonlinear effects are not sufficient to prevent the ultimate decay of the disturbance. This may be seen in figure $7(a)$, which shows the evolution of the disturbed strip when the maximum steepness predicted by linear theory, $\beta_{\mathrm{m}}$, is 0.1 . The disturbance amplifies and then decays without noticeably altering the strip-like geometry of the vortex filament. The evolution according to linear theory is shown by the dashed contours. Even at $\beta_{\mathrm{m}}=0.1$ it is apparent that nonlinearity affects the details of the evolution. It may also be seen that, although the wave slope remains small, the displacement becomes large relative to the thickness of the strip. As remarked above, this does not by itself lead to a breakdown of linear theory since the wavenumber becomes small. The prediction of the linearized long-wave equations studied in Appendix A, namely that the long-time configuration is a sinusoidally disturbed strip, with displacement increasing relative to the thickness of the strip but decaying in absolute value, is internally self-consistent.

For $\beta_{\mathrm{m}} \geqslant 0.2$ the strip rolls up into a vortex which is not extended indefinitely (consistent with Kida's 1981 description of vortices in external strain fields). The 

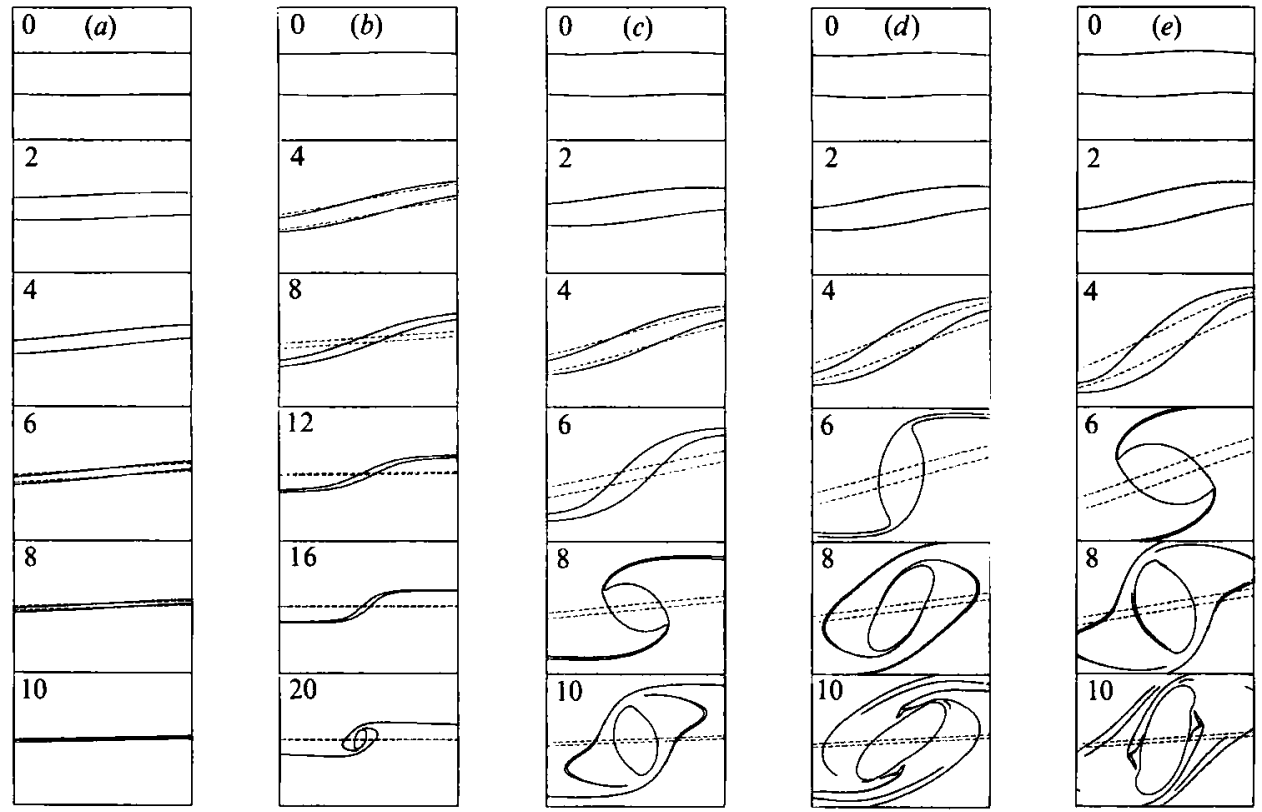

Figure 7. Fully nonlinear contour-surgery calculations of a disturbed strip of vorticity (under pure strain with $\gamma=0.05$ ). The disturbance is chosen to be the one that would amplify most in linear theory. The initial r.m.s. wave slope of the disturbance in this figure is chosen so that the maximum r.m.s. wave slope predicted by linear theory would be $(a) 0.1,(b) 0.2,(c) 0.3,(d) 0.4$ and $(e) 0.5$. The time is given in the top left-hand corner of each panel. The evolution predicted by linear theory is shown in each case by the dashed contours.

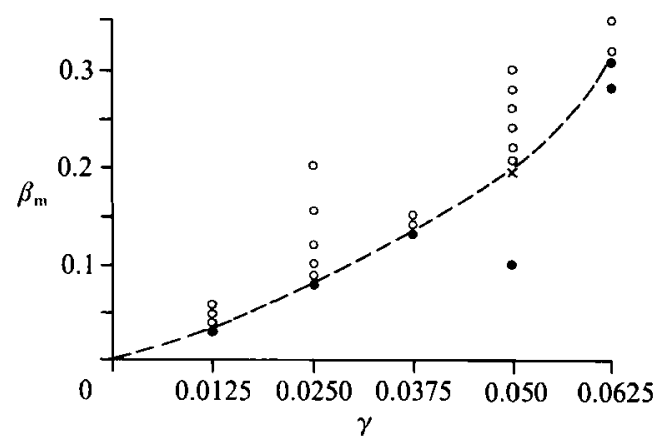

FIGURE 8. Regime diagram showing the occurrence of roll-up for various values of $\gamma$ and maximum r.m.s. wave slope $\beta_{\mathrm{m}}$ predicted by linear theory. Closed circles denote decay, open circles roll-up and the dashed line marks the transition.

evolution for $\beta_{\mathrm{m}}=0.2,0.3,0.4$ and 0.5 is shown in figure $7(b-e)$. As $\beta_{m}$ increases, the size of the vortex increases, and the time it takes to form decreases.

Computations for other values of the strain show that the critical value of $\beta_{\mathrm{m}}$ for vortex roll-up decreases as the value of the strain decreases. Figure 8 summarizes the results of a number of experiments. Solid circles correspond to decay and open circles to roll-up. The transition between decay and roll-up as a function of strain rate is shown by the dashed curve. Reference to the relevant finite-amplitude evolution equations, e.g. the generalization of (B 12) in Dritschel (1988), to the case of a strip rather than a single contour, confirms that the wave steepness is the relevant 
estimate of the importance of the nonlinear terms. It is therefore to be expected that, for all non-zero $\gamma$, the criterion for roll-up be that the steepness exceed some finite value. We conjecture that the decrease of the transition value of $\beta_{\mathrm{m}}$ with $\gamma$, apparently towards zero as $\gamma$ tends to zero, is related to the secular effects of nonlinearity over the period of amplification, which increases in duration as $-\gamma^{-1} \log \gamma$ in this limit. Some support for this explanation comes from the fact that, in the experiments conducted, the measured value of the steepness at the transition decreased less rapidly with $\gamma$ than the value of $\beta_{\mathrm{m}}$.

\section{Discussion}

There seems to be a consensus that in a number of potentially unstable flows the effect of external strain is stabilizing. These include not only the case of the twodimensional vorticity filament considered here, but also analogous three-dimensional geophysical cases where barotropic or baroclinic instabilities might have been expected. The purpose of this paper is to put this notion of stabilization by strain on a firmer quantitative footing.

The effects of uniform strain on the instability of a filament of constant vorticity have been examined. The thickness of the strip decreases in time under the strain, and a linear stability analysis is performed on this time-dependent basic flow. Strain has two principal effects on the disturbance problem: first, to compress the disturbance in the transverse direction; and second, to extend it in the longitudinal direction. The first effect is always stabilizing, while the second is ultimately so as well. All disturbances, in linear theory, eventually decay. However, there can be temporary amplification, quantified by the maximum possible increase in the r.m.s. wave slope (12). The question of how to quantify the amplification does not, of course, arise in the consideration of exponentially growing modes on a steady basic state, but seems to be crucial to any assessment of the stability of time-dependent flows. Indeed, the question has also arisen in Batchelor's (1987) study of the stability of rising bubbles; there, as here, the strain (at the forward edge of the bubble) exerts a stabilizing influence on the interface. Although that flow is steady, the author chose to analyse the behaviour of disturbances with a time-dependent (non-modal) spatial structure, and therefore had to examine the possibility of temporary growth within the context of an initial-value problem.

As in Batchelor's case it is also important to note that, since the amplification factor is finite, the initial scales and amplitudes of disturbances to the flow will play a role in determining whether or not they grow to finite amplitude (see also Moore \& Griffith-Jones 1974).

The effects of a uniform rotation of the strip relative to the background flow have also been included, for the special case in which the strip is aligned at its equilibrium orientation $\phi=\phi_{\mathrm{c}}=\frac{1}{2} \sin ^{-1}(\Omega / \gamma)$, where $\Omega$ is the rotation rate and $\gamma$ the strain rate. The stretching of the strip then occurs at a rate $\bar{\gamma}_{c}=\left(\gamma^{2}-\Omega^{2}\right)^{\frac{1}{2}}$, and there is furthermore a shear flow parallel to the strip of strength $\Lambda=-2 \Omega$. It is found that 'adverse' shear $(\Lambda>0)$ inhibits disturbance amplification while 'cooperative' shear $(\Lambda<0)$ enhances it, a result that is consistent with the findings of Dritschel $(1989 a)$ on the stabilizing effects of shear alone.

The analysis presented here has demonstrated that a stretching rate $\bar{\gamma}_{\mathrm{c}}$ exceeding $25 \%$ of the vorticity anomaly of a filament completely suppresses disturbance amplification, in the sense that the r.m.s. wave slope of disturbances can only decay with time. A stretching rate substantially less than this, about $6.5 \%$ in the case with 
$\boldsymbol{\Omega}=\mathbf{0}$ for example, is enough to prevent more than a three-fold amplification. This helps explain why the thin filaments of vorticity that arise in direct numerical simulations of two-dimensional turbulence (which exist in the presence of large-scale strain associated with coherent vortices) tend to behave quasi-passively, instead of rolling up into small vortices.

It seems possible that in the diagnosis of realistic and complicated flows, both simulated and observed, the calculation of the local stretching and shearing rates could be a useful diagnostic procedure. The relevant stretching rate is simply the rate of separation of fluid particles along a strip of vorticity, while the relevant shearing rate is twice the rate at which the strip rotates relative to the ambient strain axes, that is $A=2(\dot{\phi}-\Omega)$ (by (2) and the definition of $\Lambda$ ). For flows with continuous vorticity the stretching and shearing rates may be computed from the formulae

$$
\begin{aligned}
\bar{\gamma} & =\hat{\boldsymbol{t}} \cdot \boldsymbol{\nabla u} \cdot \hat{\boldsymbol{t}}, \\
\frac{1}{2} \boldsymbol{\Lambda} & =\hat{\boldsymbol{n}} \cdot \nabla \boldsymbol{u} \cdot \hat{\boldsymbol{t}},
\end{aligned}
$$

where $\nabla u$ is the velocity gradient tensor, $\hat{n}=\nabla \omega /|\nabla \omega|$ a unit vector parallel to the vorticity gradient, $\omega$ being the vorticity, and $\hat{\boldsymbol{t}}=-\hat{\boldsymbol{e}}_{z} \times \hat{\boldsymbol{n}}$ (a vector which points along contours of vorticity and has larger vorticity on the right). Dividing these quantities by the typical vorticity contrast across filaments, one could use the results of $\$ 4$ as an indication of those parts of the flow that would remain filamentary.

The question of whether vortex roll-up occurs is not only important for understanding idealized numerical simulations, but is also of considerable relevance to real geophysical flows, which often exhibit quasi-two-dimensional behaviour. For example, the flow in the winter hemisphere of the Earth's stratosphere appears to be divided into two regions, namely a strong polar vortex surrounded by a 'surf zone' (see e.g. Juckes \& McIntyre 1987). The two regions are of very different dynamical character and in the surf zone the motion appears to be a kind of two-dimensional turbulence. It is of crucial importance for quantifying such processes as chemical mixing to determine the nature of the turbulent motion, and in particular the extent to which it may be dominated either by thin, quasi-passive filaments of vorticity or by small-scale vortices with isolated cores. It is clear that the combined stabilizing effects of strain and shear must play a significant role in such considerations. It is possible that the quantitative results presented in this paper and their extension to spherical geometry (Dritschel \& Polvani 1991) and stratified flows (Waugh \& Dritschel 1991), can be incorporated in parametrizations of eddy transport and mixing, both in stratospheric and in other geophysical contexts.

During this work D.G. D. has been supported by the UK Science and Engineering Research Council; P.H.H. by Queens' College, Cambridge and a Royal Society Meteorological Office Research Fellowship; T.G.S. by St. Catharine's College, Cambridge, the UK Natural Environment Research Council, the Natural Sciences and Engineering Research Council of Canada and the Atmospheric Environment Service of Canada; and M.N.J. by a UK Natural Environment Research Council studentship and by NASA grant NAGW/772. Supercomputer resources were provided by SERC and the UK Universities Global Atmospheric Modelling Project and additional support was provided by the US Office of Naval Research. 


\section{Appendix A. The amplification of long-wavelength disturbances}

To study the amplification of disturbances with $k \ll 1$, for $t \sim O(1)$ it is sufficient to set $k=0$ in $(11 a, b)$ (this is not to say that the disturbances are independent of $x$ ). The equations are then independent of $A$ and the two basic solutions are given by

$$
A_{1}=1, \quad B_{1}=0 ; \quad A_{2}=-t, \quad B_{2}=1 .
$$

The first corresponds to a sinuous mode of constant amplitude, the second to an increasing sinuous mode imposed on a constant varicose mode.

The maximum amplification factor at time $t$ is

$$
\mathscr{A}^{2}=\Delta^{2}\left(1+\frac{1}{2} t^{2}+\frac{1}{2} t\left(4+t^{2}\right)^{\frac{1}{2}}\right) \text {. }
$$

The $\Delta^{2}$ factor corresponds to exponential decay, the other factor to algebraic growth; evidently decay is therefore always ultimately unavoidable (within this linear theory). Whether or not there is an initial period of temporary amplification prior to the decay is determined by the sign of $\partial \overline{\mathscr{A}} / \partial t$ at $t=0$. Using (A 2) it is readily found that $\partial \mathscr{A} / \partial t>0$ at $t=0$ if and only if

$$
-\dot{\Delta} / \Delta<\frac{1}{2}
$$

To find the maximum amplification $\mathscr{A}_{\mathrm{m}}$ and the time to maximum amplification $t_{\mathrm{m}}$ when (A 3) is satisfied, one must determine the roots of $\partial \mathscr{A} / \partial t=0$. After some algebra, one finds that $t_{\mathrm{m}}$ is a root of

$$
\left(t^{2}+4\right)^{-\frac{1}{2}}=-\dot{\Delta} / \Delta
$$

In the case of the aligned strip, where $\Delta(t)=\mathrm{e}^{-2 \bar{\gamma}_{\mathrm{c}} t}$, (A 3) indicates that amplification occurs for $\bar{\gamma}_{\mathrm{c}}<\frac{1}{4}$; otherwise all disturbances decay. When amplification does occur, the single solution to $(\mathrm{A} 4)$ is found to be

$$
\bar{\gamma}_{\mathrm{c}} t_{\mathrm{m}}=\frac{1}{2} \beta \equiv \frac{1}{2}\left(1-\left(4 \bar{\gamma}_{\mathrm{c}}\right)^{2}\right)^{\frac{1}{2}}
$$

and the maximum amplification is

$$
\mathscr{A}_{\mathrm{m}}=\mathrm{e}^{-\beta}\left(1+\frac{\beta(1+\beta)}{8 \bar{\gamma}_{\mathrm{c}}^{2}}\right)^{\frac{1}{2}},
$$

which is obtained by substituting $t$ as given by $t_{\mathrm{m}}$ in (A 5) into (A 2), together with $\Delta=\mathrm{e}^{-\beta}$. This calculation is self-consistent provided that the initial value of $k$ is small enough, even though $t_{\mathrm{m}} \gg 1$ for $\gamma_{\mathrm{c}} \ll 1$. To find the phase difference between the waves on the two interfaces, $\varphi_{\mathrm{m}} \equiv \varphi_{-}-\varphi_{+}$, for the maximal solution, one uses (A 1) and (14) to obtain

$$
\hat{\eta}_{ \pm}=\frac{1}{2}(A \mp \mathrm{i} B)=\frac{1}{2}\left\{\cos \theta-t_{\mathrm{m}} \sin \theta \mp \sin \theta\right\}
$$

(taking $\lambda_{1}=0$ and noting that $\lambda_{1}=\lambda_{2}$ for the maximal solution), whence

Then using the fact that

$$
\tan \varphi_{ \pm}=\frac{\mp \sin \theta}{\cos \theta-t_{\mathrm{m}} \sin \theta}
$$

$$
t_{\mathrm{m}}=\frac{2 \cos 2 \theta}{\sin 2 \theta}
$$


for the maximal solution, it follows that

$$
\tan \varphi_{\mathrm{m}}=\frac{2}{t_{\mathrm{m}}}
$$

Finally, in the case of non-aligned strips in a pure strain $\left(\phi_{0} \neq 0\right)$, it is necessary to determine the roots of (A 4) numerically. (See the results in figure 5, for the case $\Omega=0$.) But the criterion for amplification is nevertheless easily found from (A 3 ): using the fact that, from $(2 b), \dot{\Delta} / \Delta=-2 \gamma \cos 2 \phi_{0}$ at $t=0$, amplification obtains if and only if

$$
\gamma \cos 2 \phi_{0}<\frac{1}{4}
$$

Note that in the long-wavelength limit modes may, depending on $\Lambda$, be exponentially growing or decaying at vanishingly small rates, or have oscillatory behaviour. Neither of these can account for the amplification demonstrated above, which may be interpreted as follows. For $A<0$, the difference in structure, in the long-wave limit, between a growing mode and its decaying conjugate vanishes. An initial condition made up of equal and opposite amounts of growing and decaying modes therefore has vanishingly small amplitude. The possibility of large amplification stems from the slowly increasing difference between the two. For $A>$ 0 long-wave disturbances are neutral, but a large temporary amplification results in a similar way. The difference between rightwards-propagating and leftwardspropagating, rather than growing and decaying, modes is then the relevant quantity that vanishes in the long-wave limit.

\section{Appendix B. The weak-strain limit}

Under the condition that the strip has reached an equilibrium orientation, so that it is subject to a strain $\bar{\gamma}_{\mathrm{c}}$, it is possible to make analytical progress under the assumption that $\bar{\gamma}_{\mathrm{c}}$ is small. The first steps in the analytical calculation for $A=0$ have been made by Dhanak (1981), who exploited a limiting process in which the integrated vorticity was tacitly assumed to remain constant as the thickness of the layer tended to zero, and the vorticity jump therefore increased. An expression for the largest contribution to the amplification factor is given by Dhanak (1981, equation 3.10). Here a closely related limit is considered, in which the strain rate $\bar{\gamma}_{c}$ is the small parameter.

The relevant equations are $(11 a)$ and $(11 b)$, with $\Delta=\exp \left(-2 \bar{\gamma}_{\mathrm{e}} t\right)$. It is convenient to use $\kappa=k \Delta>0$ as the independent variable so that $(11 a)$ and $(11 b)$ reduce to

$$
-2 \bar{\gamma}_{\mathrm{c}} \kappa \frac{\mathrm{d} A}{\mathrm{~d} \kappa}=-\frac{1}{2}\left[1-\kappa(1-\Lambda)+\mathrm{e}^{-\kappa}\right] B \equiv-2 \kappa \mathscr{F}(\kappa) B
$$

and

$$
-2 \bar{\gamma}_{\mathrm{c}} \kappa \frac{\mathrm{d} B}{\mathrm{~d} \kappa}=\frac{1}{2}\left[1-\kappa(1-\Lambda)-\mathrm{e}^{-\kappa}\right] A \equiv 2 \kappa \mathscr{G}(\kappa) A
$$

Note that the evolution of the solutions as $t$ increases towards $\infty$ is followed by letting $\kappa$ decrease towards zero.

Inspection of the forms of $\mathscr{F}$ and $\mathscr{G}$ shows that for $A \geqslant 1$ both are everywhere positive in $\kappa>0$ and the solutions $A$ and $B$ are therefore oscillatory. For $A<1$ both $\mathscr{F}$ and $\mathscr{G}$ tend to $A-1$ as $\kappa \rightarrow \infty$, and $\mathscr{F}$ has a single zero at $\kappa=\kappa_{\mathscr{F}}>0$, say. For $0<\Lambda<1$ (adverse shear) $\mathscr{G}$ is positive for small $\kappa$ and has a single zero at $\kappa=\kappa_{\mathscr{G}}$, 
where $0<\kappa_{\mathscr{G}}<\kappa_{\mathscr{F}}$. Solutions of (B 1) and (B 2) are therefore oscillatory for $0<\kappa<$ $\kappa_{\mathscr{G}}$ and for $\kappa_{\mathscr{F}}<\kappa$. For $\Lambda \leqslant 0$ (cooperative shear) $\mathscr{G}$ is negative for all $\kappa$ and solutions are therefore exponential for $\kappa<\kappa_{\mathscr{F}}$ and oscillatory for $\kappa>\kappa_{\text {FF }}$.

Standard asymptotic methods may be used to solve these equations in the limit $\bar{\gamma}_{\mathrm{c}} \rightarrow 0$, although, in order to evaluate the maximum amplification factor (16) it is necessary to study in detail the form of the solution near the turning points, where one of the functions $\mathscr{F}$ or $\mathscr{G}$ is zero.

It follows from the turning-point analysis that, whatever the value of $\Lambda$, the largest amplification factor is achieved for an initial condition at

$$
\kappa=\kappa_{\mathscr{F}}+\left.1.174 \gamma_{\mathrm{c}}^{\frac{2}{3}}\left(\mathscr{F}^{\prime} \mathscr{G}\right)^{\frac{2}{3}}\right|_{\kappa_{\mathscr{G}}}
$$

where the notation $\mathscr{F}^{\prime}$ is used for $\mathrm{d} \mathscr{F} / \mathrm{d} \kappa$ (and $\mathscr{G}^{\prime}$ will be used similarly).

The resulting amplification depends on $\Lambda$ as follows.

(a) $1>A \gg \bar{\gamma}_{\mathrm{c}}$

The amplification factor, achieved at

$$
\begin{gathered}
\kappa=\kappa_{\mathscr{G}}-\left.1.019\left(-\mathscr{F} \mathscr{G}^{\prime}\right)^{\frac{2}{2}}\right|_{\kappa_{\mathcal{G}}} \frac{\gamma_{\mathrm{c}}^{2}}{}, \\
\text { is }\left.\left.\quad 1.780 \frac{\kappa_{\mathscr{G}}}{\kappa_{\mathscr{F}}} \bar{\gamma}_{\mathrm{e}}^{-\frac{1}{3}}\left(\mathscr{F} 5 \mathscr{G}^{\prime}\right)^{-\frac{1}{12}}\right|_{\kappa_{\mathcal{G}}}\left(\mathscr{F}^{\prime 7} \mathscr{G}^{\prime-1}\right)^{\frac{1}{12}}\right|_{\kappa_{\mathscr{F}}} \exp \left(\bar{\gamma}_{\mathrm{c}}^{-1} \int_{\kappa_{\mathscr{G}}}^{\kappa_{\mathscr{F}}}\left(-\mathscr{F} \mathscr{G}^{\frac{1}{2}} \mathrm{~d} \kappa^{\prime}\right) .\right.
\end{gathered}
$$

(b) $A=O\left(\bar{\gamma}_{\mathrm{c}}\right)$

The maximum amplification occurs at $\kappa=\bar{\gamma}_{\mathrm{c}} v_{0}$, where $v=v_{0}$ is the maximum of $v U\left(\frac{1}{2}-\frac{1}{4} \lambda, 1, v\right) \exp \left(-\frac{1}{4} v\right)$, where $\lambda=\bar{\gamma}_{\mathrm{c}}^{-1} \Lambda$ and $U$ is the confluent hypergeometric function defined by Abramowitz \& Stegun (1960, equation 13.1.6). Given $\lambda$ the corresponding value of $v$ may be evaluated numerically. The maximum amplification factor is given by

$$
\begin{aligned}
\left.0.937 \kappa_{\mathscr{F}}^{-1}\left(\mathscr{F}^{\prime} \mathscr{G}\right)^{-\frac{1}{12}}\right|_{\kappa_{\mathscr{F}}} \max _{v>0} & \left\{v U\left(\frac{1}{2}-\frac{\lambda}{4}, 1, v\right) \exp \left(-\frac{1}{4} v\right)\right\} \\
& \times \bar{\gamma}_{\mathrm{c}}^{\frac{1}{8}} \mathrm{e}^{\frac{1}{4} \mu \lambda}\left(2 \bar{\gamma}_{\mathrm{c}} \kappa_{\mathscr{F}}\right)^{\frac{1}{4} \lambda} \exp \left(\bar{\gamma}_{\mathrm{c}}^{-1} \int_{0}^{\kappa_{\mathscr{F}}}(-\mathscr{F} \mathscr{G})^{\frac{1}{2}} \mathrm{~d} \kappa^{\prime}\right),
\end{aligned}
$$

where

$$
\mu=\int_{0}^{\kappa_{F}}\left[\frac{1-\kappa^{\prime}}{\left[\mathrm{e}^{-2 \kappa^{\prime}}-\left(1-\kappa^{\prime}\right)^{2}\right]^{\frac{1}{2}}}-\frac{1}{\kappa^{\prime}}\right] \mathrm{d} \kappa^{\prime} .
$$

$\mathscr{F}, \mathscr{G}$ and associated quantities are to be defined in (B $4 a$ ) and (B $4 b$ ) with $A=0$. The value of the integral appearing inside the last exponential in (B $4 a$ ) is 0.188 .

(c) $-\Lambda \gg \bar{\gamma}_{\mathrm{c}}$

The maximum amplification is attained at $\kappa=2 \bar{\gamma}_{\mathrm{c}}^{2} \hat{v}_{0} / \Lambda$, where $\hat{v}=\hat{v}_{0}$ is the argument for which the function $\hat{v}^{2} K_{0}(\hat{v})$ achieves its maximum, where $K_{0}$ is the modified Bessel function defined by Abramowitz \& Stegun (1960, equation 9.6.13). It follows that the maximum amplification factor is given by

$$
\left.0.721 \kappa_{\mathscr{F}}^{-1}\left(\mathscr{F}^{\prime 7} \mathscr{G}\right)^{-\frac{1}{12}}\right|_{\kappa_{\mathscr{F}}} \bar{\gamma}_{\mathrm{c}}^{-\frac{4}{3}} \exp \left(\bar{\gamma}_{\mathrm{c}}^{-1} \int_{0}^{\kappa_{\mathscr{F}}}(-\mathscr{F} \mathscr{G})^{\frac{1}{2}} \mathrm{~d} \kappa^{\prime}\right) .
$$

It may be shown that the expression (B 5) agrees with (B 4a) in the limit $\lambda \rightarrow \infty$.

Some indication of the smallness of $\bar{\gamma}_{\mathrm{c}}$ required for the expressions (B 3b), (B 4a) 
and (B 5) to be numerically accurate may be gained from inspection of the turning region at $\kappa=\kappa_{\mathscr{F}}$. For $A=0$ for example, $\kappa_{\mathscr{F}} \approx 1.28, \mathscr{F}^{\prime}=0.154$ and $\mathscr{G}=-0.119$. Thus the width of the turning region, as defined by the rescaling, is $16.3 \frac{2}{\gamma_{\mathrm{e}}^{3}}$, equal to 0.75 when $\gamma=0.01$ and 0.163 when $\bar{\gamma}_{\mathrm{c}}=0.001$. It therefore appears that $\bar{\gamma}_{\mathrm{c}} \leqslant 0.001$ is a likely requirement for the asymptotic estimate of the amplification factor to be in good numerical agreement with the actual value.

\section{REFERENCES}

Abramowitz, M. \& Stegun, I. 1960 Handbook of Mathematical Functions. US National Bureau of Standards.

Babiano, A., Baspevant, C., Legras, B. \& Sadourny, R. 1987 Vorticity and passive-tracer dynamics in two-dimensional turbulence. J. Fluid Mech. 183, 379-397.

BatchelOR, G. K. 1987 The stability of a large gas bubble rising through liquid. J. Fluid Mech. $184,399-422$.

Brachet, M. E., Meneguzzi, M., Politano, H. \& Sulem, P. L. 1988 'The dynamics of freely decaying two-dimensional turbulence. . I. Fluid Mech. 194, 333-349.

Dhanak, M. R. 1981 The stability of an expanding circular vortex layer. Proc. R. Soc. Lond. A $375,443-451$.

Dritschel, D. G. 1988 The repeated filamentation of two-dimensional vorticity interfaces. J. Fluid Mech. 194, 511-547.

Dritschel, D. G. $1989 a$ On the stabilization of a two-dimensional vortex strip by adverse shear. J. Fluid Mech. 206, 193-221.

Dritschel, D. G. $1989 b$ Contour dynamics and contour surgery: numerical algorithms for extended high-resolution modelling of vortex dynamics in two-dimensional, inviscid, incompressible flows. Comput. Phys. Rep. 10, 77-146.

Dritscher, D. G. 1990 The stability of elliptical vortices in an external straining flow. J. Fluid Mech. 210, 223-261.

Dritschal, D. G. \& Polvani, L. M. 1991 The roll-up of vortex strips on a sphere. J. Fluid Mech. (in press).

Juckes, M. N. \& McIntYRe, M. E. 1987 A high-resolution, one-layer model of breaking planetary waves in the stratosphere. Nature 328, 590-596.

KIDA, S. 1981 Motion of an elliptic vortex in a uniform shear flow. J. Phys. Soc. Japan 50, $3517-3520$.

Legras, B., Santangelo, P. \& Benzi, R. 1988 High-resolution numerical experiments for forced two-dimensional turbulence. Europhys. Lett. 5, 37-42.

McWilliams, J.C. 1984 The emergence of isolated coherent vortices in turbulent flow. J. Fluid Mech. 146, 21-43.

Moore, D. W. 1976 The stability of an evolving two-dimensional vortex sheet. Mathematika 23, 128-133.

Moore, D. W. \& Griffith-Jones, R. 1974 The stability of an expanding circular vortex sheet. Mathematika 21, 128-133.

Moore, D. W. \& Saffman, P. G. 1971 Structure of a line vortex in an imposed strain. In Aircraft Wake Turbulence and its Detection (ed. J. H. Olsen, A. Goldburg \& M. Rogers), pp. 339-354. New York: Plenum.

MOORE, D. W. \& Saffman, P. G. 1975 The instability of a straight vortex filament in a strain field. Proc. $R$. Soc. Lond. A 346, 413-425.

RAYleigh, LoRd 1880 On the stability, or instability, of certain fluid motions. Proc. Lond. Math. Soc. 11, 57-70. (Also in Rayleigh's Theory of Sound, Vol. 2, §368, Dover, 1945.)

RAYLEIGH, LoRd 1887 On the stability, or instability, of certain fluid motions, II. Proc. Lond. Math. Soc. 19, 67-74. (Also in Rayleigh's Theory of Sound, Vol. 2, §367, Dover, 1945.)

Santangelo, R., Benzi, R. \& Legras, B. 1989 The generation of vortices in high-resolution, twodimensional decaying turbulence and the influence of initial conditions on the breaking of selfsimilarity. Phys. Fluids A 1, 1027-1034. 
Shelley, M. J. \& BAKER, G. R. 1990 On the connection between thin vortex layers and vortex sheets. J. Fluid Mech. 215, 161-194.

Tsai, C.-Y. \& Widnall, S. E. 1976 The stability of short waves on a straight vortex filament in a weak externally imposed strain field. J. Fluid Mech. 73, 721-733.

WAUGH, D. W. \& Dritschel, D. G. 1991 The stability of filamentary vorticity in twodimensional geophysical vortex dynamics models J. Fluid Mech. 231, 575-598. 